\title{
Outcomes of oesophageal cancer treated with neoadjuvant compared with definitive chemoradiotherapy
}

Caryn Wujanto ${ }^{1}$ MRCP , Jeremy Tey ${ }^{1}{ }_{F R A N C Z R}$, Balamurugan Vellayappan ${ }^{1}{ }_{F R A N C Z R}$, Jimmy $\underline{\operatorname{So}}^{2}{ }_{F R C S}$, Wei Peng Yong $^{3}{ }_{M R C P}$, Asim $\underline{\text { Shabbir }^{2}}{ }_{\text {FRCS }}$, Michelle Tseng ${ }^{1}{ }_{\text {FRANCZR, Yu Yang Soon }}{ }^{1}$ FRANCZR, Francis $\underline{\text { Ho }}{ }^{1}{ }_{\text {FRANCZR }}$

\begin{abstract}
Introduction: We report outcomes of patients with oesophageal cancer treated with neoadjuvant chemoradiotherapy (NACRT) plus surgery or definitive chemoradiotherapy (chemoRT) at our institution.

Methods: We retrospectively reviewed patients who underwent chemoRT from 2005 to 2017. The primary outcome was overall survival (OS). Secondary outcomes were disease-free survival (DFS) and toxicities.

Results: We identified 96 patients with median age of 64 years and squamous cell carcinoma in $82.3 \%$. Twenty-nine patients $(30.2 \%)$ received NACRT plus surgery, 67 patients $(69.8 \%)$ received definitive chemoRT. Median follow-up was 13.5 months. The 3/5-year OS were 26.4\%/13.4\%, and $59.6 \% / 51.6 \%$ in the definitive chemoRT and NACRT plus surgery groups, respectively. The 3/5-year DFS were $19.3 \% / 12.3 \%$, and $55.7 \% / 37.2 \%$ in the definitive chemoRT and NACRT plus surgery groups, respectively. NACRT plus surgery significantly improved OS (hazard ratio [HR] 0.40, 95\% confidence interval [CI] $0.22-0.72, P<0.01$ ) and DFS (subhazard ratio [SHR] 5.21, 95 CI 1.20-22.7, $P=0.03$ ). Multivariable analysis for OS in the definitive chemoRT group indicated stage (1-2 vs $3-4 \mathrm{a}$; HR $2.17,95 \%$ CI $1.15-4.11, P=0.02)$ and feeding tube (no tube versus tube; HR $1.85,95 \%$ CI $1.00-3.43, P=0.05)$ as significantly associated with OS. The cumulative incidence of local recurrence was significantly higher in the definitive chemoRT group (SHR 5.21, 95 CI 1.2022.7, $P=0.03)$. Nineteen patients $(65.5 \%)$ had postoperative complications.

Conclusion: NACRT plus surgery improved OS and DFS. However, in view of treatment-related complications, careful selection of patients is warranted. With the predominant histology of our cohort being squamous cell carcinoma (SCC), our results may be more relevant for those with SCC.
\end{abstract}

Ann Acad Med Singap 2021;50:536-47

Keywords: Neoadjuvant chemoradiotherapy, oesophageal cancer, surgery

\section{INTRODUCTION}

Oesophageal cancer is the 7 th most common cancer in the world, with 572,034 new cases diagnosed in $2018,{ }^{1}$ and is the 6th most common cause of cancerrelated mortality worldwide. ${ }^{2}$ Oesophageal squamous cell carcinoma (SCC) is the predominant histological subtype. However, the incidence of adenocarcinoma has risen among the Western population due to the rising prevalence of central obesity and gastrooesophageal reflux disease. ${ }^{3-5}$
Studies have reported high recurrence and mortality rates from oesophageal cancer, hence the use of multimodality treatment to improve survival. ${ }^{6-8}$ The ChemoRadiotherapy for Oesophageal cancer followed by Surgery Study (CROSS) randomised controlled trial reported long-term survival benefit with neoadjuvant chemoradiotherapy (NACRT) plus surgery in resectable locally advanced oesophageal SCC/adenocarcinoma when compared to surgery alone. ${ }^{9}$ However, other studies showed comparable survival with definitive

\footnotetext{
${ }^{1}$ Department of Radiation Oncology, National University Cancer Institute, Singapore

${ }^{2}$ Division of Surgical Oncology, National University Cancer Institute, Singapore

${ }^{3}$ Department of Haematology-Oncology, National University Cancer Institute, Singapore

Correspondence: Dr Jeremy Tey, Department of Radiation Oncology, 1E Kent Ridge Road, Level 7, NUHS Tower Block, Singapore 119228.

Email: jeremy_tey@nuhs.edu.sg
} 


\section{CLINICAL IMPACT}

\section{What is New}

- This study provides outcomes of patients with oesophageal cancer treated with neoadjuvant chemoradiotherapy followed by surgery or definitive chemoradiotherapy at an academic medical centre in Singapore.

- Patients treated with neoadjuvant chemoradiotherapy plus surgery had improved overall survival and disease-free survival.

\section{Clinical Implications}

- This study supports the use of neoadjuvant chemoradiotherapy followed by surgery for patients with localised oesophageal cancer. However, careful selection of patients is warranted.

chemoradiotherapy (chemoRT) alone, particularly in SCC. ${ }^{10,11}$

For locally advanced SCC and adenocarcinoma (cT1b-cT2 $\mathrm{N}+$ or cT3-cT4aN), the latest National Comprehensive Cancer Network (NCCN) guideline recommends NACRT in surgically fit patients with non-cervical oesophageal SCC or definitive chemoRT for cervical oesophageal SCC. ${ }^{12}$ For those with cT2N0 SCC or adenocarcinoma, NCCN recommends oesophagectomy for low-risk lesions $(<3 \mathrm{~cm}$, well differentiated) and NACRT or definitive chemoRT for those with high-risk lesions (lymphovascular invasion, $\geq 3 \mathrm{~cm}$, poorly differentiated). ${ }^{12}$

In this study, we evaluate the outcome of patients in our institution treated with NACRT plus surgery versus definitive chemoRT for locally advanced oesophageal SCC and adenocarcinoma.

\section{METHODS}

This study at the National University Cancer Institute, Singapore was approved by the National Healthcare Group Domain Specific Review Board Domain B.

\section{Patients}

We conducted a retrospective review of patients with histologically confirmed oesophageal carcinoma who underwent curative intent chemoRT $+/$ - surgery at our institution from 2005 to 2017. Tumour staging was based on the American Joint Cancer Committee 8th edition Cancer Staging Manual. ${ }^{13}$ Patients who received prior definitive, neoadjuvant or palliative intent RT were excluded.

\section{Treatment}

\section{Radiotherapy}

All patients received radiotherapy (RT) with 3dimensional conformal or intensity modulated RT (IMRT). RT was delivered in $1.8 \mathrm{~Gy}$ daily fractions (50.4Gy in 28 fractions for definitive RT, 41.4-50.4Gy in 23-28 fractions as per the CROSS trial for neoadjuvant $\mathrm{RT}^{9}$ ) with $10 \mathrm{MV}$ photon beams, 5 days/week. As per our department protocol, the clinical target volume (CTV) included the gross tumour volume (GTV) with $3 \mathrm{~cm}$ margin superiorly/inferiorly, $0.5 \mathrm{icm}$ margin in the axial dimension. The planning target volume (PTV) included the CTV with $1 \mathrm{~cm}$ margin. Elective nodal coverage includes bilateral supraclavicular nodes for tumours above the carina and celiac axis coverage for distal oesophageal tumours $(32-40 \mathrm{~cm}$ from incisors). Lung dose is limited to V20Gy $<35 \% /$ mean lung dose $<18 \mathrm{~Gy}$, heart dose V40Gy $<30 \%$ /mean heart dose $<26$ Gy, spinal cord $<45$ Gy max dose.

\section{Chemotherapy}

Chemotherapy regimens include paclitaxel/carboplatin or fluorouracil/oxaliplatin for neoadjuvant or definitive chemoRT with the alternative of fluorouracil/cisplatin for definitive regimen. As per NCCN guideline: intravenous paclitaxel $50 \mathrm{mg} / \mathrm{m}^{2}$, intravenous carboplatin area under curve (AUC) 2, given on day 1 and weekly thereafter for 5 weeks. Intravenous oxaliplatin $85 \mathrm{mg} / \mathrm{m}^{2}$, leucovorin $400 \mathrm{mg} / \mathrm{m}^{2}$, intravenous fluorouracil $400 \mathrm{mg} / \mathrm{m}^{2}$ push, fluorouracil $800 \mathrm{mg} / \mathrm{m}^{2}$ continuous infusion on days 1 and 2 , are given every 2 weeks for a total of 3 cycles concurrently with RT. ${ }^{12}$

\section{Surgery}

Suitability for surgery and type of resection depends on the tumour location, anatomy and surgeons' preference. Surgical approaches include Ivor Lewis oesophagogastrectomy (laparotomy + right thoracotomy), McKeown oesophago-gastrectomy (right thoracotomy + laparotomy + cervical anastomosis) and transhiatal oesophagogastrectomy (laparotomy + cervicalanastomosis). ${ }^{12}$

\section{Data collection}

Data obtained from our institution's medical records and RT databases include clinical diagnosis, RT technique/dose, chemotherapy, treatment break/ completion, surgical procedures, and complications. 
Follow-up data were obtained from patients' medical record up until the time of death or most recent review.

\section{Performance status, comorbidities and overall health status}

Performance status was assessed using the Eastern Cooperative Oncology Group (ECOG) grading. ${ }^{14}$ The extent of comorbid conditions was evaluated using the Charlson Comorbidity Index. ${ }^{15}$ The International Society of Geriatric Oncology (SIOG) score was used for patients $\geq 65$ years to assess their functional status, and pre-existing comorbidities. ${ }^{16}$

\section{Follow-up}

Clinical examination (with endoscopy and imaging if clinically indicated) was scheduled 3-6 monthly for the first 2 years, 6-12 monthly for the 3rd to 5 th year, and annually thereafter. ${ }^{12}$

\section{Outcomes}

Overall survival (OS) was defined as the time from the first treatment to death due to any cause. Diseasefree survival (DFS) was defined as the time from the first treatment to the time of first recurrence (local or distant) as detected by endoscopy or imaging. Local recurrence was defined as the time from the first treatment to the time of local recurrence detected by endoscopy or imaging.

\section{Toxicity}

Toxicities were graded according to the Common Terminology Criteria for Adverse Events (CTCAE) version $5.0 .^{17}$

\section{Statistical analysis}

OS and DFS were estimated using the Kaplan-Meier method. Survival differences were compared using the $\log$ rank test. Univariable and multivariable cox proportional hazard regression models were performed to identify independent factors with significant impact on survival. Factors analysed include: age group $(<64$ vs $\geq 65$ ), sex (men vs women), stage (stage $1 / 2$ vs $3 / 4 a$ ), weight loss (no vs yes), surgery (no vs yes), feeding tube (no vs yes), T-stage, histology (SCC vs adenocarcinoma), RT (IMRT vs 3DCRT), RT break (no vs yes), chemotherapy break (no vs yes), Charlson score, SIOG score and ECOG grading. Factors with a $P$ value of $\leq 0.2$ on univariable analyses were entered into the multivariable model. Competing risk regression with death as a competing risk was performed to compare local recurrence rates between the NACRT plus surgery versus definitive chemoRT groups.

Chi-square test was used to compare baseline characteristics between the NACRT plus surgery and definitive chemoRT groups.

Statistical analysis was performed using STATA version 14 (StataCorp LLC, College Station, US). A $P$ value of $\leq 0.05$ was considered to indicate statistical significance.

\section{RESULTS}

\section{Patient characteristics}

Ninety-six patients underwent curative RT for oesophageal carcinoma at our institution (Table 1). Majority were men (87.5\%). Median age was 64 years (range $30-88$ years).

\section{Tumour characteristics}

All had histological diagnosis of oesophageal carcinoma $(82.3 \% \mathrm{SCC})$. Majority of tumours were within the thoracic oesophageal region (58.3\%), mostly stages 2 and 3 (45.8\% and 36.5\%, respectively). The predominate depth of tumour invasion as detected on CT scan was T3 (51 patient, 53.1\%) and most patients had no nodal involvement $(45.8 \%)$ while 41 patients (42.7\%) had N1 disease (Table 1).

\section{Treatment}

\section{Radiotherapy}

All patients received RT; 67 patients (69.8\%) received definitive RT and 29 patients (30.2\%) received NACRT. Most received 50.4Gy/28 fractions for definitive RT (74.6\%). As for NACRT, $48.3 \%$ received $41.4 \mathrm{~Gy} / 23$ fractions, $48.3 \%$ received $50.4 \mathrm{~Gy}$ in 28 fractions.

RT break (i.e. number of days that a patient did not have RT) ranged from 1-12 days and was recorded in 31 patients (32.3\%), mostly within the definitive chemoRT group (Table 2). Reasons include fever, mucositis, diarrhoea, dehydration, stent insertion, bleeding, fatigue, stroke, fall and machine repair. Majority (93 patients, 96.9\%) completed RT.

\section{Chemotherapy}

Eighty-four patients $(87.5 \%)$ received concurrent chemotherapy with intravenous paclitaxel/carboplatin in 38 patients (45.2\%) and intravenous fluorouracil with leucovorin plus platinum-based chemotherapy (cisplatin or oxaliplatin) in 20 patients $(23.8 \%)$. Other regimens include oral capecitabine $\left(625 \mathrm{mg} / \mathrm{m}^{2}\right.$ twice a day on days $1-5$ every week for 5 weeks) plus intravenous 


\begin{tabular}{|c|c|c|c|c|}
\hline & $\begin{array}{c}\text { All }(\mathrm{N}=96) \\
\text { No. }(\%)\end{array}$ & $\begin{array}{c}\text { No surgery (67) } \\
\text { No. }(\%)\end{array}$ & $\begin{array}{l}\text { Surgery (29) } \\
\text { No. (\%) }\end{array}$ & $\chi^{2} P$ value \\
\hline \multicolumn{5}{|l|}{ Sex } \\
\hline Male & $84(87.5)$ & $59(88.1)$ & $25(86.2)$ & \\
\hline Female & $12(12.5)$ & $8(11.9)$ & $4(13.8)$ & 0.80 \\
\hline \multicolumn{5}{|l|}{ Age } \\
\hline$<64$ & $54(56.3)$ & $34(50.7)$ & $20(69.0)$ & \\
\hline$\geq 65$ & $42(43.8)$ & $33(49.3)$ & $9(31.0)$ & 0.10 \\
\hline \multicolumn{5}{|l|}{ Ethnicity } \\
\hline Chinese & $79(82.3)$ & $56(83.6)$ & $23(79.3)$ & \\
\hline Indian & $6(6.3)$ & $4(6.0)$ & $2(6.9)$ & \\
\hline Malay & $4(4.2)$ & $2(3.0)$ & $2(6.9)$ & \\
\hline Others & $7(7.3)$ & $5(7.5)$ & $2(6.9)$ & 0.84 \\
\hline \multicolumn{5}{|l|}{ ECOG } \\
\hline 0 & $22(22.9)$ & $8(11.9)$ & $14(48.3)$ & \\
\hline 1 & $68(70.8)$ & $55(82.1)$ & $13(44.8)$ & \\
\hline 2 & $5(5.2)$ & $3(4.5)$ & $2(6.9)$ & $<0.01$ \\
\hline 3 & $1(1.0)$ & $1(1.5)$ & 0 & \\
\hline \multicolumn{5}{|c|}{ Charlson score } \\
\hline $0-1$ & $69(71.9)$ & 48 (71.6) & $21(72.4)$ & \\
\hline $2-3$ & $22(22.9)$ & $16(23.9)$ & $6(20.7)$ & \\
\hline $4-6$ & $5(5.2)$ & $3(4.5)$ & $2(6.9)$ & 0.85 \\
\hline \multicolumn{5}{|c|}{ SIOG Group } \\
\hline 1 & $69(71.9)$ & $47(70.1)$ & $22(75.9)$ & \\
\hline 2 & $13(13.5)$ & $10(14.9)$ & $3(4.5)$ & \\
\hline 3 & $2(2.1)$ & $2(3.0)$ & 0 & 0.53 \\
\hline \multicolumn{5}{|l|}{ CT staging } \\
\hline $\mathrm{T} 1$ & $2(2.1)$ & 0 & $2(6.9)$ & \\
\hline $\mathrm{T} 2$ & $28(29.2)$ & $22(32.8)$ & $6(20.7)$ & \\
\hline $\mathrm{T} 3$ & $51(53.1)$ & $33(49.3)$ & $18(62.1)$ & \\
\hline $\mathrm{T} 4$ & $15(15.6)$ & $12(17.9)$ & $3(10.3)$ & 0.31 \\
\hline No & $44(45.8)$ & $36(53.7)$ & $8(27.6)$ & \\
\hline N1 & $41(42.7)$ & $29(43.3)$ & $12(41.4)$ & \\
\hline $\mathrm{N} 2$ & $10(10.4)$ & $2(3.0)$ & $8(27.6)$ & $<0.01$ \\
\hline $\mathrm{Nx}$ & $1(1.0)$ & 0 & $1(3.4)$ & \\
\hline
\end{tabular}

oxaliplatin ( $85 \mathrm{mg} / \mathrm{m}^{2}$ on days 1,15 and 29 for 3 doses). Twenty-six patients $(31 \%)$ required chemotherapy break (range 1-14 days) due to neutropenic fever, thrombocytopenia, pneumonia, dehydration and haematemesis. Fifty-one patients $(60.7 \%)$ completed all planned cycles of chemotherapy. 
Table 1. Patients and tumour characteristics (Cont'd)

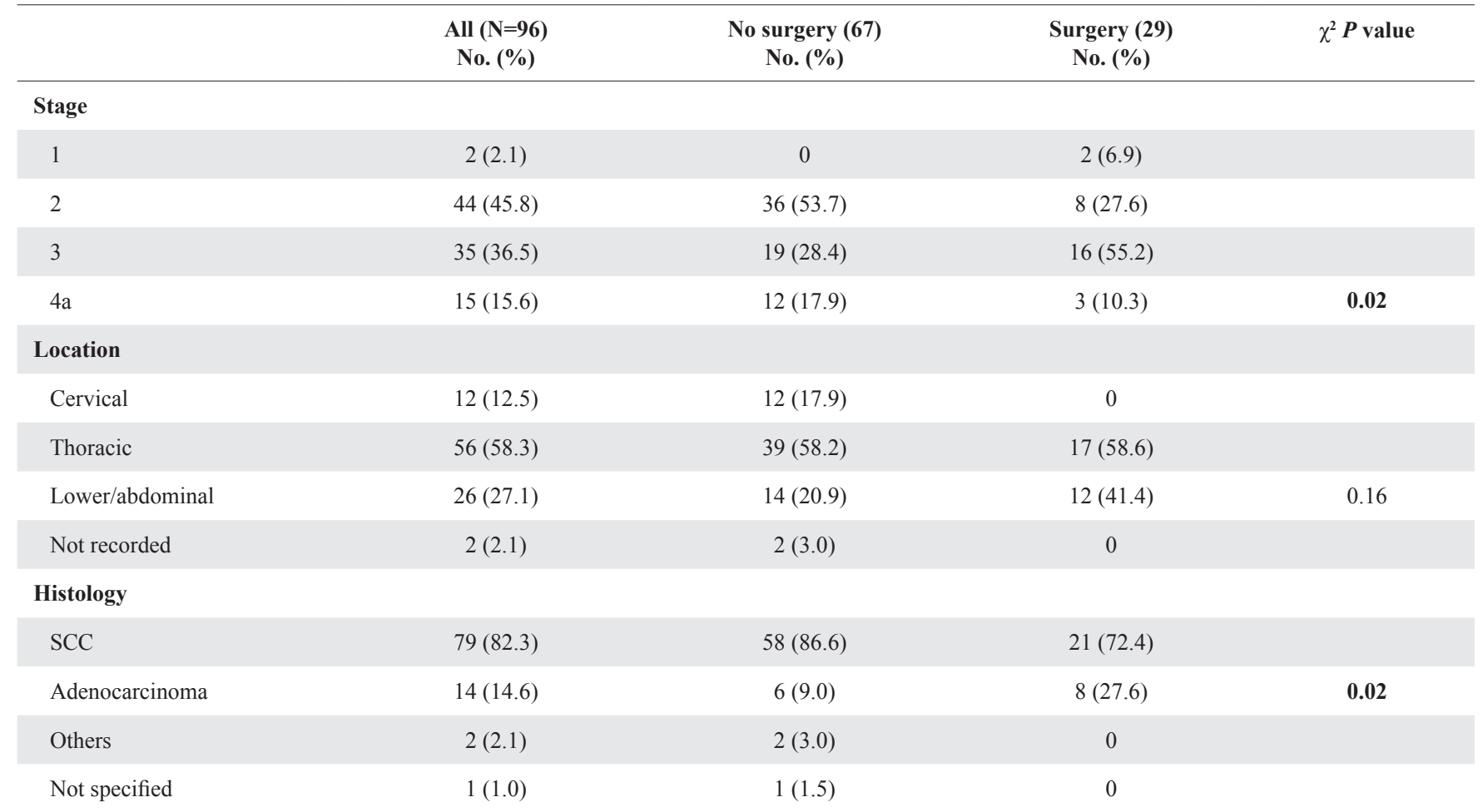

CT: computed tomography; ECOG: Eastern Cooperative Oncology Group; SIOG: International Society of Geriatric Oncology: SCC: squamous cell carcinoma

$P$ values in bold are significant

\section{Surgery}

Twenty-nine patients (30.2\%) had surgery. Median time between completion of RT to surgery was 50 days (range 17-109). Twenty-three patients underwent Ivor-Lewis oesophagogastrectomy, 1 patient underwent transhiatal oesophagogastrectomy, and 5 had various approaches (McKeown oesophagogastrectomy, oesophagectomy with jejunal transposition, total oesophagectomy, total gastrectomy, and Ivor Lewis oesophagogastrectomy with total cholecystectomy). One patient (3.4\%) had a positive proximal margin (radial/distal margins clear). Pathologic complete response was achieved in 9 patients $(31.0 \%)$. Downstaging of the tumour $(\mathrm{T})$ stage was achieved in 18 patients $(62.1 \%)$, and downstaging of the lymph node $(\mathrm{N})$ stage was achieved in 11 patients $(37.9 \%)$ (Table 3$)$.

\section{Cause of death}

Fifty-four patients (56.3\%) died during the period. Out of 37 patients with recorded cause of death, 24 patients died of advanced oesophageal cancer. Twelve patients died of pneumonia and 1 died of acute myocardial infarction. Two patients died of postoperative complications and referred to the coroners.

\section{Outcomes and subgroup analyses}

\section{Overall Survival}

Median follow-up was 13.5 months (range 1-132). Median OS for all patients was 18 months. Median OS was 71 months (range 3-114) and 13 months (range 1-132) for the NACRT plus surgery, and definitive chemoRT groups, respectively. The 3- and 5-year OS were $26.4 \%$ and $13.4 \%$, respectively, in patients who underwent definitive chemoRT, and 59.6\% and 51.6\%, respectively, in patients who underwent NACRT plus surgery (Table 2). Patients treated with NACRT plus surgery had significantly improved OS compared to patients treated with definitive chemoRT (hazard ratio [HR] $0.40,95 \%$ confidence interval [CI] $0.22-$ $0.72, P<0.01)$. There was no significant difference in OS between tumours located within the cervical region and other locations (HR0.99, 95\% CI 0.47-2.09, $P<0.985$ ) (Fig. 1).

In the 29 patients who had NACRT plus surgery, higher radiation dose improved survival. Fourteen patients who received $50.4 \mathrm{~Gy} / 28$ fractions due to borderline resectable tumours had significantly improved survival compared to 14 patients who 
Table 2. Treatment characteristics, comparison of overall survival, overall survival by stage and disease-free survival between patients with and without surgery

\begin{tabular}{|c|c|c|c|c|}
\hline & $\begin{array}{c}\text { All }(\mathrm{N}=96) \\
\text { No. }(\%)\end{array}$ & $\begin{array}{c}\text { No surgery (67) } \\
\text { No. }(\%)\end{array}$ & $\begin{array}{l}\text { Surgery (29) } \\
\text { No. }(\%)\end{array}$ & $\chi^{2} P$ value \\
\hline \multicolumn{5}{|l|}{ Feeding tube } \\
\hline Nasogastric & $12(12.5)$ & $9(13.4)$ & $3(10.3)$ & \\
\hline Nasojejunal & $15(15.6)$ & $11(16.4)$ & $4(13.8)$ & \\
\hline None & $61(63.5)$ & $40(59.7)$ & $21(72.4)$ & 0.58 \\
\hline \multicolumn{5}{|l|}{ Stenting } \\
\hline Yes & $11(11.5)$ & $10(14.9)$ & $1(3.4)$ & \\
\hline No & $85(88.5)$ & $57(85.1)$ & $28(96.6)$ & 0.11 \\
\hline \multicolumn{5}{|l|}{ RT technique } \\
\hline 3DCRT & $33(34.4)$ & $27(40.3)$ & $6(20.7)$ & \\
\hline IMRT & $63(65.6)$ & $40(59.7)$ & $23(79.3)$ & 0.06 \\
\hline \multicolumn{5}{|l|}{ RT dose } \\
\hline 41.4Gy/23\# & $15(15.6)$ & $1(1.5)$ & $14(48.3)$ & \\
\hline $41.7 \mathrm{~Gy} / 25 \#$ & $1(1.0)$ & $1(1.5)$ & 0 & \\
\hline $45 \mathrm{~Gy} / 25 \#$ & $1(1.0)$ & $1(1.5)$ & 0 & \\
\hline $48 \mathrm{~Gy} / 24 \#$ & $1(1.0)$ & $1(1.5)$ & 0 & \\
\hline $50 \mathrm{~Gy} / 25 \#$ & $3(3.1)$ & $2(3.0)$ & 1 & \\
\hline $50.4 \mathrm{~Gy} / 28 \#$ & $64(66.7)$ & $50(74.6)$ & $14(48.3)$ & $<0.01$ \\
\hline $56 \mathrm{~Gy} / 28 \#$ & $1(1.0)$ & $1(1.5)$ & 0 & \\
\hline $66 \mathrm{~Gy} / 33 \#$ & $2(2.1)$ & $2(3.0)$ & 0 & \\
\hline $66.6 \mathrm{~Gy} / 37 \#$ & $1(1.0)$ & $1(1.5)$ & 0 & \\
\hline \multicolumn{5}{|l|}{ RT break } \\
\hline Yes & $31(32.3)$ & $22(32.8)$ & $9(3.1)$ & \\
\hline No & $58(60.4)$ & $38(56.7)$ & $20(69.0)$ & 0.60 \\
\hline Not recorded & $7(7.3)$ & 0 & $7(2.4)$ & \\
\hline \multicolumn{5}{|c|}{ Concurrent chemo } \\
\hline Yes & $84(87.5)$ & $55(82.1)$ & $29(43.3)$ & \\
\hline No & $4(4.2)$ & $4(6.0)$ & 0 & \\
\hline Not recorded & $8(8.3)$ & $8(11.9)$ & 0 & \\
\hline \multicolumn{5}{|l|}{ Type of surgery } \\
\hline Ivor-Lewis & 23 & 0 & $23(79.3)$ & \\
\hline Transhiatal & 1 & 0 & $1(3.4)$ & \\
\hline Others & 5 & 0 & $5(17.2)$ & \\
\hline
\end{tabular}


Table 2. Treatment characteristics, comparison of overall survival, overall survival by stage and disease-free survival between patients with and without surgery (Cont'd)

\begin{tabular}{|c|c|c|c|c|}
\hline & All (N=96) & No surgery (67) & Surgery (29) & $\chi^{2} P$ value \\
\hline \multicolumn{5}{|l|}{ Overall survival } \\
\hline Median, months & 18 & 13 & & 71 \\
\hline Range, months & $1-132$ & $1-132$ & & $3-114$ \\
\hline $1 \mathrm{yr}(\%)$ & & 50.6 & & 75.0 \\
\hline 3 yr (\%) & & 26.4 & & 59.6 \\
\hline $5 \mathrm{yr}(\%)$ & & 13.4 & & 51.6 \\
\hline \multicolumn{5}{|c|}{ Overall survival by stage } \\
\hline $\begin{array}{l}\text { Stage } 2 \\
1 \text { yr }(\%) \\
3 \text { yr }(\%) \\
5 \text { yr }(\%)\end{array}$ & & $\begin{array}{l}58.8 \\
45.7 \\
23.2\end{array}$ & & $\begin{array}{l}100 \\
85.7 \\
57.1\end{array}$ \\
\hline $\begin{array}{l}\text { Stage } 3 \\
1 \text { yr (\%) } \\
3 \text { yr (\%) } \\
5 \text { yr (\%) }\end{array}$ & & $\begin{array}{c}45.1 \\
5.6 \\
5.6\end{array}$ & & $\begin{array}{l}60.0 \\
45.7 \\
45.7\end{array}$ \\
\hline $\begin{array}{l}\text { Stage } 4 \\
1 \text { yr (\%) } \\
3 \text { yr (\%) } \\
5 \text { yr (\%) }\end{array}$ & & $\begin{array}{l}35.2 \\
11.7 \\
11.7\end{array}$ & & $\begin{array}{l}66.7 \\
33.3 \\
33.3\end{array}$ \\
\hline Disease-free survi & & & & \\
\hline $1 \mathrm{yr}(\%)$ & & 35.1 & & 71.4 \\
\hline 3 yr $(\%)$ & & 19.3 & & 55.7 \\
\hline 5 yr $(\%)$ & & 12.3 & & 37.2 \\
\hline
\end{tabular}

3DCRT: 3-dimensional radiotherapy; IMRT: intensity modulated radiotherapy; PEG: percutaneous endoscopic gastrostomy; RT: radiotherapy

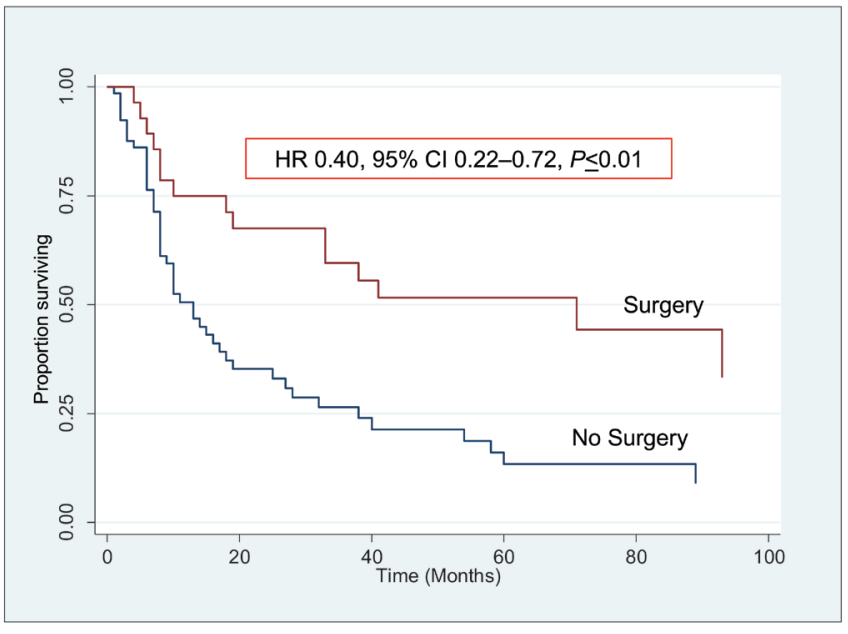

Fig. 1. Kaplan Meier survival curves of patients who had surgery versus no surgery.

HR: hazard ratio; $\mathrm{CI}$ : confidence interval

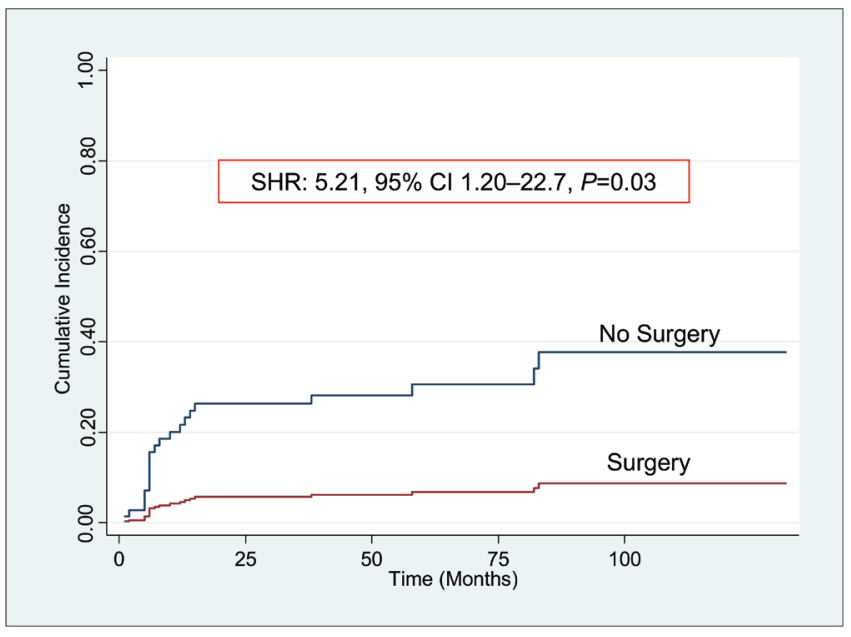

Fig. 2. Cumulative incidence of local recurrence (surgery versus no surgery).

SHR: subhazard ratio; CI: confidence interval 
Table 3. Pre- and postoperative TNM staging of patients $(n=29)$

\begin{tabular}{|c|c|c|c|}
\hline $\begin{array}{l}\text { Preoperative } \\
\text { TNM stage }\end{array}$ & No. (\%) & $\begin{array}{l}\text { Postoperative } \\
\text { TNM stage }\end{array}$ & No. $(\%)$ \\
\hline \multirow[t]{5}{*}{$\mathrm{T} 1$} & $2(6.9)$ & T0 & $2(100)$ \\
\hline & & $\mathrm{T} 1$ & $0(0)$ \\
\hline & & $\mathrm{T} 2$ & $0(0)$ \\
\hline & & $\mathrm{T} 3$ & $0(0)$ \\
\hline & & $\mathrm{T} 4$ & $0(0)$ \\
\hline \multirow[t]{6}{*}{$\mathrm{T} 2$} & $6(20.7)$ & T0 & $1(16.7)$ \\
\hline & & $\mathrm{T} 1$ & $3(50)$ \\
\hline & & $\mathrm{T} 2$ & $0(0)$ \\
\hline & & $\mathrm{T} 3$ & $0(0)$ \\
\hline & & $\mathrm{T} 4$ & $0(0)$ \\
\hline & & Unknown & $2(33.3)$ \\
\hline \multirow[t]{6}{*}{$\mathrm{T} 3$} & $18(62.1)$ & T0 & $1(5.6)$ \\
\hline & & $\mathrm{T} 1$ & $4(22.2)$ \\
\hline & & $\mathrm{T} 2$ & $2(11.1)$ \\
\hline & & $\mathrm{T} 3$ & $8(44.4)$ \\
\hline & & $\mathrm{T} 4$ & $0(0)$ \\
\hline & & unknown & $3(16.7)$ \\
\hline \multirow[t]{5}{*}{$\mathrm{T} 4$} & $3(10.3)$ & T0 & $1(33.3)$ \\
\hline & & $\mathrm{T} 1$ & $0(0)$ \\
\hline & & $\mathrm{T} 2$ & $0(0)$ \\
\hline & & $\mathrm{T} 3$ & $2(66.7)$ \\
\hline & & $\mathrm{T} 4$ & $0(0)$ \\
\hline \multirow[t]{4}{*}{ N0 } & 8 (27.6) & No & $5(62.5)$ \\
\hline & & N1 & $1(12.5)$ \\
\hline & & N2 & $0(0)$ \\
\hline & & unknown & $2(25)$ \\
\hline \multirow[t]{4}{*}{ N1 } & $12(41.4)$ & N0 & $4(33.3)$ \\
\hline & & N1 & $5(41.7)$ \\
\hline & & N2 & $2(16.7)$ \\
\hline & & unknown & $1(8.3)$ \\
\hline \multirow[t]{4}{*}{ N2 } & 8 (27.6) & N0 & $4(50)$ \\
\hline & & N1 & $2(25)$ \\
\hline & & N2 & $1(12.5)$ \\
\hline & & unknown & $1(12.5)$ \\
\hline $\mathrm{Nx}$ & $1(3.4)$ & No & $1(100)$ \\
\hline
\end{tabular}

TNM: tumour, node, metastasis received lower dose RT with $41.4 \mathrm{~Gy} / 23$ fractions (HR 5.36, 95\% CI 1.46-19.75, $P<0.012$ ).

Multivariable analysis for OS in the definitive chemoRT group showed that stage (1-2 vs $3-4 \mathrm{a}$; HR $2.40,95 \%$ CI $1.30-4.44, P<0.01)$ was the only significant variable and in the NACRT group, stage (1-2 vs 3-4a; HR $4.26,95 \%$ CI $1.19-15.22, P=0.03$ ) and RT dose (41.4 Gy vs $>50.4 \mathrm{~Gy}$; HR 4.63, 95\% CI 0.97-22.0, $P=0.05$ ) were significant (Table 4).

\section{Disease-free survival}

The 3- and 5-year DFS were $19.3 \%$ and $12.3 \%$, respectively, in patients who underwent definitive chemoRT, and $55.7 \%$ and $37.2 \%$ in patients who underwent NACRT plus surgery.

Multivariable analysis for DFS in patients treated with definitive chemoRT showed that age group $(<64$ vs $\geq 65$ years; HR $0.66,95 \%$ CI $0.37-1.17, P=0.16$ ), stage (1-2 vs 3-4a; HR $1.92,95 \%$ CI $1.07-3.43$, $P=0.03$ ), feeding tube (no tube vs tube; HR $2.36,95 \%$ CI 1.29-4.32, $P<0.01$ ), histology (SCC vs adenocarcinoma/others; HR $0.66,95 \%$ CI $0.37-1.17, P=0.16$ ) were significant and in patients treated with NACRT, RT break (no break vs break; HR 4.00, 95\% CI $1.26-12.70, P=0.02$ ) was the only significant variable associated with DFS.

\section{Local recurrence}

Twenty-two patients $(22.9 \%)$ had local recurrence, of which 2 patients had NACRT plus surgery and 20 patients had definitive chemoRT. Ten were detected following clinical presentation, 5 were detected through computed tomography (CT) scan, 4 through positron emission tomography-CT scan, and 3 through upper gastrointestinal endoscopy. Local recurrence occurred within the high dose RT region in all 20 cases, with 1 case also having a recurrence within the marginal region. The cumulative incidence of local recurrence was significantly higher in patients treated with definitive chemoRT compared to NACRT plus surgery (subhazard ratio [SHR] 5.21, 95 CI 1.20-22.7, $P=0.03$ ) (Fig. 2).

\section{Distant recurrence}

Thirty-seven patients $(38.5 \%)$ had distant recurrence, of which 11 patients had NACRT plus surgery, 26 patients only had definitive chemoRT. The most common sites were in the lung (12 patients), lymph nodes ( 7 patients), brain (6 patients), bone (3 patients) and liver (3 patients). 
Table 4. Univariable and multivariable analysis for overall survival in definitive and preoperative chemoradiotherapy

\begin{tabular}{|c|c|c|c|}
\hline \multicolumn{4}{|c|}{ Definitive ChemoRT - Univariable analysis for overall survival } \\
\hline Variable & HR & $95 \%$ CI & $P$ \\
\hline $\begin{array}{l}\text { Age group } \\
<64 \text { (ref) vs } \geq 65\end{array}$ & 0.55 & $0.31-0.99$ & 0.049 \\
\hline $\begin{array}{l}\text { Sex } \\
\text { Male (ref) vs female }\end{array}$ & 1.11 & $0.44-2.83$ & 0.82 \\
\hline ECOG & 1.20 & $0.71-2.02$ & 0.51 \\
\hline $\begin{array}{l}\text { Stage } \\
\text { Stage } 1-2 \text { (ref) vs 3-4a }\end{array}$ & 2.17 & $1.20-3.91$ & 0.01 \\
\hline $\begin{array}{l}\text { Weight loss } \\
\text { No (ref) vs yes }\end{array}$ & 1.17 & $0.85-1.61$ & 0.33 \\
\hline $\begin{array}{l}\text { Feeding tube } \\
\text { No (ref) vs yes }\end{array}$ & 1.91 & $1.06-3.46$ & 0.03 \\
\hline $\begin{array}{l}\text { Histology } \\
\text { SCC (ref) vs adeno/others }\end{array}$ & 1.61 & $0.75-3.44$ & 0.22 \\
\hline T-stage & 1.27 & $0.80-2.03$ & 0.31 \\
\hline $\begin{array}{l}\text { RT dose } \\
\geq 50.4 \text { Gy vs }<50.4 \text { Gy (ref) }\end{array}$ & 1.20 & $0.47-3.08$ & 0.70 \\
\hline $\begin{array}{l}\text { RT technique } \\
\text { IMRT (ref) vs 3DCRT }\end{array}$ & 1.35 & $0.75-2.44$ & 0.31 \\
\hline $\begin{array}{l}\text { RT break } \\
\text { Yes (ref) vs No }\end{array}$ & 0.85 & $0.45-1.59$ & 0.61 \\
\hline Charlson comorbidity score & 1.08 & $0.82-1.27$ & 0.87 \\
\hline SIOG score & 0.91 & $0.52-1.61$ & 0.76 \\
\hline Variable & HR & $95 \% \mathrm{CI}$ & $\boldsymbol{P}$ \\
\hline $\begin{array}{l}\text { Age group } \\
<64 \text { (ref) vs } \geq 65\end{array}$ & 0.57 & $0.31-1.06$ & 0.08 \\
\hline $\begin{array}{l}\text { Stage } \\
\text { Stage } 1-2 \text { (ref) vs } 3-4 a\end{array}$ & 2.40 & $1.30-4.44$ & $<0.01$ \\
\hline $\begin{array}{l}\text { Feeding tube } \\
\text { No (ref) vs yes }\end{array}$ & 1.76 & $0.96-3.24$ & 0.07 \\
\hline \multicolumn{4}{|c|}{ Neoadjuvant ChemoRT - Univariable analysis for overall survival } \\
\hline Variable & HR & $95 \% \mathrm{CI}$ & $P$ \\
\hline $\begin{array}{l}\text { Age group } \\
<64 \text { (ref) vs } \geq 65\end{array}$ & 1.35 & $0.46-3.98$ & 0.59 \\
\hline $\begin{array}{l}\text { Gender } \\
\text { Male (ref) vs female }\end{array}$ & 0.36 & $0.48-2.78$ & 0.33 \\
\hline ECOG & 1.44 & $0.69-2.97$ & 0.33 \\
\hline $\begin{array}{l}\text { Stage } \\
\text { Stage } 1-2 \text { (ref) vs } 3-4 a\end{array}$ & 2.27 & $0.72-7.17$ & 0.16 \\
\hline $\begin{array}{l}\text { Weight loss } \\
\text { No (ref) vs yes }\end{array}$ & 0.81 & $0.38-1.71$ & 0.58 \\
\hline $\begin{array}{l}\text { Feeding tube } \\
\text { No (ref) vs yes }\end{array}$ & 0.88 & $0.24-3.19$ & 0.84 \\
\hline $\begin{array}{l}\text { Histology } \\
\text { SCC (ref) vs adeno/others }\end{array}$ & 1.86 & $0.62-5.57$ & 0.27 \\
\hline
\end{tabular}


Table 4. Univariable and multivariable analysis for overall survival in definitive and preoperative chemoradiotherapy (Cont'd)

\begin{tabular}{|c|c|c|c|}
\hline \multicolumn{4}{|c|}{ Neoadjuvant ChemoRT - Univariable analysis for overall survival } \\
\hline Variable & HR & $95 \% \mathrm{CI}$ & $P$ \\
\hline T stage & 1.31 & $0.68-2.54$ & 0.42 \\
\hline $\begin{array}{l}\text { RT dose } \\
41.4 \mathrm{~Gy} \text { vs } \geq 50.4 \mathrm{~Gy} \text { (ref) }\end{array}$ & 5.92 & $1.60-21.90$ & 0.01 \\
\hline $\begin{array}{l}\text { RT technique } \\
\text { IMRT (ref) vs 3DCRT }\end{array}$ & 0.16 & $0.02-1.35$ & .09 \\
\hline $\begin{array}{l}\text { RT break } \\
\text { Yes (ref) vs No }\end{array}$ & 0.25 & $0.09-0.74$ & 0.01 \\
\hline Charlson score & 0.85 & $0.26-2.77$ & 0.78 \\
\hline SIOG score & 0.79 & $0.10-6.17$ & 0.82 \\
\hline \multicolumn{4}{|c|}{ Multivariable (include univariable with cut-off at 0.2 ) } \\
\hline Variable & HR & $95 \% \mathrm{CI}$ & $\boldsymbol{P}$ \\
\hline $\begin{array}{l}\text { Stage } \\
\text { Stage } 1-2 \text { (ref) vs } 3-4 a\end{array}$ & 4.26 & $1.19-15.22$ & 0.03 \\
\hline $\begin{array}{l}\text { RT break } \\
\text { Yes (ref) vs No }\end{array}$ & 0.34 & $0.09-1.26$ & 0.09 \\
\hline $\begin{array}{l}\text { RT dose } \\
41.4 \mathrm{~Gy}_{-} \mathrm{vs} \geq 50.4 \mathrm{~Gy}\end{array}$ & 4.63 & $0.97-22.0$ & 0.05 \\
\hline $\begin{array}{l}\text { RT technique } \\
\text { IMRT (ref) vs 3DCRT }\end{array}$ & 0.68 & $0.06-7.84$ & 0.76 \\
\hline
\end{tabular}

3DCRT: 3-dimensional radiotherapy; adeno: adenocarcinoma; CI: confidence interval; HR: hazard ratio; IMRT: intensity modulated radiotherapy; RT: radiotherapy; SCC: squamous cell carcinoma; SIOG: International Society of Geriatric Oncology

$P$ values in bold are significant

\section{Toxicity}

\section{Acute toxicity of definitive chemoRT}

Most patients tolerated the treatment. Five patients experienced at least grade 3 toxicity ( 3 cases of tracheoesophageal fistula, 1 case of grade 3 mucositis with resulting percutaneous endoscopic gastrostomy tube insertion, 1 case of grade 3 dermatitis). One out of 5 patients with grade 3 toxicity did not complete chemoRT due to tracheoesophageal fistula and stopped treatment after $34 \mathrm{~Gy} / 17$ fractions and 2 cycles of chemotherapy.

One patient developed sealed perforation of the gastro-oesophageal junction tumour 10 days after chemoRT and had septic shock. He recovered, underwent total gastrectomy, developed post-op intra-abdominal sepsis secondary to anastomotic leak requiring a laparotomy. He died 41 days after the initial surgery.

\section{Late toxicity of definitive chemoRT}

One patient developed radiation pneumonitis and recovered. Two patients developed oesophageal stricture, with one requiring multiple dilatations and the other a nasogastric tube insertion.

\section{Postoperative complications}

Nineteen patients $(65.5 \%)$ developed postoperative complications which included: anastomotic leak (6 patients), pneumonia (6 patients), pneumothorax (5 patients), pleural effusion (4 patients), sepsis (3 patients), fistula ( 2 patients), wound infection (1 patient), and perforation (1 patient).

\section{DISCUSSION}

In our study of 96 patients with oesophageal carcinoma, we found that patients who underwent NACRT plus surgery had significantly improved OS compared to 
definitive chemoRT alone. The NACRT plus surgery group had more patients with ECOG 0 compared to the definitive group ( $48.3 \%$ vs $11.9 \%)$. Interestingly, although there were 2 patients with stage 1 disease in the NACRT plus surgery group, there were fewer patients with stage 2 disease and more patients with stage 3 disease in the NACRT plus surgery group, compared to the definitive chemoRT group (stage 2: $27.6 \%$ vs $53.7 \%$; stage $3: 55.2 \%$ vs $28.4 \%$ ). Among those who had surgery, higher dose of RT (50.4Gy/28 fractions) significantly improved survival compared to lower dose of RT (41.4Gy/23 fractions). DFS was also better in the NACRT plus surgery compared to definitive chemoRT alone group. Our study showed that RT break was significantly associated with DFS. As RT break prolongs the total duration of RT, this results in less favourable outcome due to accelerated repopulation of tumour cells. ${ }^{18}$ In our cohort, significantly higher proportion of patients with adenocarcinoma underwent surgery compared to SCC. This may be due to previous randomised controlled trials on patients with predominantly oesophageal SCC reporting limited or no survival benefit with surgery. ${ }^{10,11}$

Our findings are consistent with studies reporting improved survival with NACRT plus surgery. A retrospective study on 298 patients from the Asian population with oesophageal SCC comparing neoadjuvant versus definitive chemoRT reported improved outcome with NACRT plus esophagectomy (HR of death $0.56,95 \%$ CI $0.42-0.75, P<0.001$ ). ${ }^{19}$ In another retrospective study on Asian patients, Wong et al. reported an estimated median survival of 24.2 months vs 12.7 months $(P=0.047)$ in 46 patients from the "CROSS eligible" group and 42 patients in the "CROSS ineligible" group, respectively. ${ }^{20}$ We reported a median OS of 71 months and 13 months for the NACRT plus surgery group and definitive chemoRT group, respectively. Our data are more similar to the CROSS trial that reported a median survival of 81.6 months in the NACRT plus surgery group. ${ }^{9}$ Hofstetter et al. reported significant improvement in OS with NACRT (3-year OS 56\% with NACRT vs $34 \%$ with no NACRT, $P=0.003)$ with higher likelihood of a complete resection being achieved..$^{21}$

In our study, patients who had NACRT plus surgery had significantly lower local recurrence compared to definitive chemoRT alone. Hofstetter et al. showed that those receiving NACRT plus surgery also had significantly fewer locoregional recurrence compared to those who did not $(17 \%$ vs $25 \%, P=0.01) .{ }^{21}$ These results are consistent with a Cochrane systematic review that showed a reduction in local recurrence with the addition of surgery to chemoRT. ${ }^{22}$

However, not all studies have reported benefit in OS with NACRT plus surgery. The abovementioned Cochrane systematic review reported that the addition of esophagectomy only provided little or no difference in OS (HR 0.99, 95\% CI 0.79-1.24, $P=0.92, \mathrm{I}^{2}=0 \%$, 2 trials) and may even be associated with higher treatment-related mortality. ${ }^{22}$ One of the studies included in the review was a randomised controlled trial comparing neoadjuvant vs definitive chemoRT in 444 patients with thoracic oesophageal SCC. There was no survival benefit with surgery (2-year OS $34 \%$ in NACRT vs $40 \%$ in definitive chemoRT only; HR $0.90, P=0.44) .{ }^{11}$ The other study in the review found that the addition of surgery improved local control but not OS in patients with SCC treated with chemoradiotherapy. ${ }^{10}$ For oesophageal adenocarcinoma, a sequential prospective non-randomised phase II studies on 35 patients also reported comparable outcome between NACRT and definitive chemoRT. ${ }^{23}$

On multivariable analysis for OS, we found that there was no significant difference for OS in SCC compared to adenocarcinoma. This is in keeping with other studies who have also not found significant difference in outcome based on histology. Interestingly, one study noted a shift in the predominant histology of oesophageal cancer among the Western population during their study period from SCC to adenocarcinoma (29\% adenocarcinoma in $1970-1985 ; 83 \%$ adenocarcinoma in 1997-2001), which was also accompanied by a shift in the location of the tumour from the upper/ mid-oesophageal region to the lower/gastro-oesophageal junction. ${ }^{20} \mathrm{~A}$ retrospective study by Tustumi et al. reported no significant difference in OS between SCC and adenocarcinoma. They reported a 5 -year OS of $22.8 \%$ in patients with SCC vs $20.2 \%$ in patients with adenocarcinoma. In their cohort of patients treated with curative intent surgical resection, 5-year OS was 56.6\% in SCC and 28\% in adenocarcinoma. ${ }^{24}$ Eloubeidi et al. reported age at diagnosis, race, lower oesophageal tumour, and increasing depth of invasion as factors associated with increased mortality risk in oesophageal carcinoma and tumour length, number, as well as proportion of lymph nodes as important prognostic factors in oesophageal carcinoma. ${ }^{25}$

The strengths of our study are that patients were treated using a standardised treatment with quality 
assurance for RT performed within a week of commencing treatment. Limitations include its retrospective nature and the relatively small patient numbers, most of whom had SCC. Selection bias might have occurred in that surgery were offered to fitter patients; there could be further selection bias for those who received a higher dose of RT ( $\geq 50 \mathrm{~Gy})$ and that good responders had subsequent surgery. In addition, as this was a non-randomised comparison, there may have been confounders that were not accounted for and reviewer bias may have led to underreporting of treatment toxicities.

\section{CONCLUSION}

In this study, we report outcomes comparable to internationally published data. Our results suggest that NACRT plus surgery reduced local recurrences and improved OS; however, careful selection of patient is warranted to minimise perioperative risks. With the predominant histology of our cohort being SCC, results from our study may be more relevant for SCCs within the Asian population.

\section{REFERENCES}

1. Bray F, Ferlay J, Soerjomataram I, et al. Global cancer statistics 2018: GLOBOCAN estimates of incidence and mortality worldwide for 36 cancers in 185 countries. CA Cancer J Clin 2018;68:394-424.

2. Torre LA, Bray F, Siegel RL, et al. Global cancer statistics, 2012. CA Cancer J Clin 2015;65:87-108

3. Hur C, Miller M, Kong CY, et al. Trends in esophageal adenocarcinoma incidence and mortality. Cancer 2013;119:1149-58.

4. Kubo A, Corley DA. Body mass index and adenocarcinomas of the esophagus or gastric cardia: a systematic review and meta-analysis. Cancer Epidemiol Biomarkers Prev 2006;15:872-8.

5. Singh S, Sharma AN, Murad MH, et al. Central adiposity is associated with increased risk of esophageal inflammation, metaplasia, and adenocarcinoma: a systematic review and meta-analysis. Clin Gastroenterol Hepatol 2013;11:1399-412.e7.

6. Bosset JF, Gignoux M, Triboulet JP, et al. Chemoradiotherapy followed by surgery compared with surgery alone in squamous-cell cancer of the esophagus. N Engl J Med 1997;337:161-7.

7. Burmeister BH, Smithers BM, Gebski V, et al. Surgery alone versus chemoradiotherapy followed by surgery for resectable cancer of the oesophagus: a randomised controlled phase III trial. Lancet Oncol 2005;6:659-68.

8. Saeki H, Morita M, Nakashima Y, et al. Neoadjuvant chemoradiotherapy for clinical stage II-III esophageal squamous cell carcinoma. Anticancer Res 2011;31:3073-7.
9. Shapiro J, van Lanschot JJB, Hulshof MCCM, et al. Neoadjuvant chemoradiotherapy plus surgery versus surgery alone for oesophageal or junctional cancer (CROSS): long-term results of a randomised controlled trial. Lancet Oncol 2015;16:1090-8.

10. Stahl M, Stuschke M, Lehmann N, et al. Chemoradiation with and without surgery in patients with locally advanced squamous cell carcinoma of the esophagus. J Clin Oncol 2005;23:2310-7.

11. Bedenne L, Michel P, Bouché O, et al. Chemoradiation followed by surgery compared with chemoradiation alone in squamous cancer of the esophagus: FFCD 9102. J Clin Oncol 2007;25:1160-8.

12. National Comprehensive Cancer Network. Esophageal and Esophagogastric Junction Cancers. Available at: https://www.ncen. org. Accessed on 27 May 2020.

13. American Joint Committee on Cancer Staging System, 2018. Available at: https://www.cancerstaging.org/references-tools/Pages/ What-is-Cancer-Staging.aspx. Accessed on 27 May 2020.

14. ECOG Performance Status, 2020. Available at: https://ecog-acrin.org/ resources/ecog-performance-status. Accessed on 27 May 2020.

15. Sundararajan V, Henderson T, Perry C, et al. New ICD-10 version of the Charlson comorbidity index predicted in-hospital mortality. J Clin Epidemiol 2004;57:1288-94.

16. Droz JP, Aapro M, Balducci L, et al. Management of prostate cancer in older patients: updated recommendations of a working group of the International Society of Geriatric Oncology. Lancet Oncol 2014;15:e404-14

17. National Institutes of Health, National Cancer Institute. Common Terminology Criteria for Adverse Events (CTCAE) version 5.0, 2017. Available at: https://ctep.cancer.gov/protocolDevelopment/ electronic_applications/docs/CTCAE_v5_Quick_Reference_8.5x11. pdf. Accessed on 27 May 2020.

18. Trott KR. Cell repopulation and overall treatment time. Int J Radiat Oncol Biol Phys 1990;19:1071-5.

19. Chen CY, Li CC, Chien CR. Neoadjuvant vs definitive concurrent chemoradiotherapy in locally advanced esophageal squamous cell carcinoma patients. World J Surg Oncol 2018;16:141.

20. Wong IYH, Lam KO, Chan W, et al. Real-world Scenario: CROSS Regimen as Preoperative Therapy for Oesophageal Squamous Cell Carcinoma. J Gastrointest Surg 2020;24:1937-47

21. Hofstetter W, Swisher SG, Correa AM, et al. Treatment outcomes of resected esophageal cancer. Ann Surg 2002; 236:376-85.

22. Vellayappan BA, Soon YY, Ku GY, et al. Chemoradiotherapy versus chemoradiotherapy plus surgery for esophageal cancer. Cochrane Database Syst Rev 2017;8:CD010511.

23. Algan O, Coia LR, Keller SM, et al. Management of adenocarcinoma of the esophagus with chemoradiation alone or chemoradiation followed by esophagectomy: results of sequential nonrandomized phase II studies. Int J Radiat Oncol Biol Phys 1995;32:753-61.

24. Tustumi F, Kimura CM, Takeda FR, et al. Prognostic factors and survival analysis in esophageal carcinoma. Arq Bras Cir Dig 2016;29:138-41.

25. Eloubeidi MA, Desmond R, Arguedas MR, et al. Prognostic factors for the survival of patients with esophageal carcinoma in the U.S.: the importance of tumor length and lymph node status. Cancer 2002;95:1434-43. 